\title{
The Implication of Candida Infection in Oral Lichen Planus Lesions
}

\author{
Ioanina PARLATESCUa, Carmen NICOLAEa, Serban TOVARUa, Lavinia RADU ${ }^{b}$,
} Ovidiu PENESc, Valentin VARLAS ${ }^{d}$

aOral Medicine Department, Faculty of Dental Medicine, "Carol Davila" University of Medicine and Pharmacy, Bucharest, Romania

b"Carol Davila" University of Medicine and Pharmacy, Bucharest, Romania

'Department of Anesthesiology and Intensive Care, University Clinical Hospital, "Carol Davila" University of Medicine and Pharmacy, Bucharest, Romania

'Department of Obstetrics and Gynecology, Faculty of Dental Medicine,

"Carol Davila" University of Medicine and Pharmacy, Bucharest, Romania

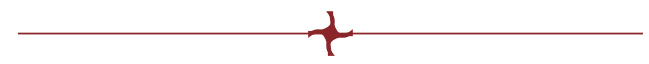

\begin{abstract}
Objective: The purpose of this study is to determine if superimposed infection with Candida species in oral lichen planus exacerbates the clinical symptoms and lesions.

Material and methods: We conducted a retrospective study on the medical charts of oral lichen planus $(O L P)$ patients diagnosed by clinical and histopathological criteria. A baseline demographic, anamnestic, clinical data, results of the oral candida investigations were analyzed and recorded for all patients.

Results: From a total number of 203 OLP patients, oral candidiasis was diagnosed in 75 (36.94\%) cases. There was no statistical significance between the clinical forms of lichen planus and the presence of Candida infection. Of all encountered Candida species, Candida albicans was most commonly detected, followed by Candida krusei, Candida lusitaniae, and Candida tropicalis.

Conclusions: Although our study is limited regarding the sample size and no statistically significant difference has been found between clinical features of OLP and oral candidiasis, a routine screening of OLP patients for oral candidiasis and a preventive antifungal strategy in the OLP treatment schedule is needed.
\end{abstract}

Keywords: oral lichen planus, oral candidiasis, oral mucosa, Candida infection.

Address for correspondence:

Ovidiu PENES

Tel.: +40 743043 043; email: ovidiu.penes@umfcd.ro

\section{INTRODUCTION}

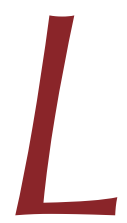

ichen planus is an autoimmune disease with chronic evolution and repeated flare-ups episodes. It involves both the skin or nails and the oral, genital, or esophageal mucosa (1). The incidence and prevalence of oral lichen planus (OLP) were analyzed through various observational and de- scriptive studies, both globally and at the level of some population subgroups. The results of these researches are quite different, depending on selection criteria, heterogeneity of study groups, and number of patients included in research. A recent meta-analysis (2) that included the most representative epidemiological studies on OLP by demographic criteria showed a prevalence of 
$0.89 \%$ among the general population and $0.98 \%$ among in-hospital patients. Also, with regard to the geographical distribution of OLP, there was a slightly higher prevalence in European countries $(1.68 \%)$ compared to research conducted on groups of patients from Asian countries (0.57\%) or South America (1.39\%). Furthermore, this meta-analysis showed a higher prevalence of OLP in females (1.69\%) compared to males $(1.09 \%)$ as well as in adults over 40 years of age $(1.90 \%)$ when compared to younger groups (0.62\%) (2).

During evolution, the OLP clinical appearance is polymorphic and dynamic, and only in sporadic cases there is a unique clinical type of lesions $(3,4)$. However, the unanimously currently accepted clinical criteria include the presence of reticular keratosis lesions located bilaterally on the posterior third of the buccal mucosa $(5,6)$. Confirmation of the clinical diagnosis by a histopathological examination is most often necessary when there is polymorphism of the lesions. A thorough differential diagnosis must be made with other diseases of the oral mucosa. Moreover, a close correlation was observed between the clinical form and the histological features. In addition to confirming the clinical diagnosis, histopathological examination provides important information about the degree of histological activity of oral lichen planus and excludes epithelial dysplasia (4). Being a chronic disease with a long evolution, OLP can cause complications, and the most serious is developing an oral carcinoma. In fact, OLP is included in the group of oral diseases at risk of malignancy (7). Other less severe complications are the association of oral candidiasis or appearance of submucosal fibrosis (1). Oral candidiasis is the most common infection that occurs in the oral cavity. Thus, in almost half of patients, Candida albicans is part of the oral cavity normal flora. Local and general imbalances can lead to uncontrolled Candida multiplication and the appearance of fungal infection. Oral Candida pathogens may colonize, invade, and induce mucosal lesions in both immunocompetent and immunocompromised individuals. Among the genus Candida, the species albicans is the most frequently associated with oral infections. Then, in order of frequency, other species may also be involved in the etiology of oral candidiasis, such as Candida glabrata, Candida krusei, or Candida tropicalis. Depending on whether or not candidiasis overlaps with other oral mucosal diseases, it is divided into two types: primary and secondary. The mucosal defense against Candida is cell-mediated immunity ( $T$ cells), and also macrophages, neutrophils, and cytotoxic T cells, and OLP shows high levels of T and B cells (8). Thus, OLP is one of the diseases often associated with oral candidiasis, especially non-albicans species. It is very important to investigate a possible fungal superimposed infection in OLP lesions because symptoms and clinical appearance may aggravate. To avoid therapeutic failure and resistant species selection, oral candidiasis therapy is established by the antifungal susceptibility testing (9).

The purpose of this study is to determine if superimposed infection with Candida species in oral lichen planus exacerbates the clinical symptoms and lesions.

\section{MATERIAL AND METHODS}

retrospective chart of records of oral lichen planus adult patients was conducted in the clinical department of Oral Pathology Discipline, Faculty of Dental Medicine, "Carol Davila" University of Medicine and Pharmacy, Bucharest, Romania. These consecutively OLP patients were diagnosed from January 2015 to October 2018 based on clinical and histopathological criteria. Baseline demographic, anamnestic, medical, and clinical data were recorded for all patients. The chief complaint was classified as pain, burning sensation, discomfort and referral by other dental or medical practitioners. Regarding the medical history, a particular emphasis was given to other diseases frequently associated with OLP such as hepatitis C, autoimmune thyroiditis, diabetes, and other autoimmune diseases. If symptoms were present before the first consultation, we divided as follows: under six months, between six months to one year, between one year to five years, and more than five years. The site of the oral lesions and the predominant clinical form (keratotic, atrophic, erosive-ulcerative, and bulous) were recorded. Results of the mycological investigation (established by culture) and detection of Candida species were analyzed and correlated with the symptoms and clinical form of the disease. Cases with incomplete data were excluded from the present study. Signed informed consent was obtained from all patients. Preliminary data of the current 
research were previously presented as a poster to the Congress of "Carol Davila" University of Medicine and Pharmacy, Bucharest, Romania (10). Fisher's test was used to determine the association between Candida infection and the clinical form of OLP.

\section{RESULTS}

total of 203 patients were included in the present study. The majority of them were females $(154,75.86 \%)$, and $49(24.13 \%)$ males. The average age was 56.75 years old, ranging from 20 to 88 years. Table 1 shows the main characteristics of patients, including the medical history, anamnestic data, and clinical features of the OLP patients. The most frequent oral lesions location was buccal mucosa (53 cases-26.10\%) followed closely by buccal mucosa and tongue (52 cases-25.61\%). Keratotic tongue lesions with and without superimposed fungal infection are presented in Figure 1.

From the total number of OLP patients, 75 (36.94\%) presented a positive mycological exam. Table 2 shows the distribution of clinical forms of lichen planus according to the presence of candidiasis infection. As a frequency of species, Candida albicans was identified as the most

\section{Characteristics}

Chief complaint

Associated medical history

Duration of symptoms until the first consultation

Site of lesions

\section{Number of cases}

\begin{tabular}{|l|l|}
\hline Pain & $38(18.71 \%)$ \\
\hline Burning sensation & $69(33.99 \%)$ \\
\hline Discomfort & $17(8.37 \%)$ \\
\hline $\begin{array}{l}\text { Referred to by other } \\
\text { dental or medical } \\
\text { practitioner }\end{array}$ & $53(26.10 \%)$ \\
\hline
\end{tabular}

\begin{tabular}{|l|l|}
\hline Autoimmune thyroiditis & $38(18.71 \%)$ \\
\hline Cutaneous lichen planus & $32(15.76 \%)$ \\
\hline Diabetes & $16(7.88 \%)$ \\
\hline Hepatitis C & $8(3.94 \%)$ \\
\hline
\end{tabular}

\begin{tabular}{|l|l|}
\hline Under six months, & $70(34.48 \%)$ \\
\hline $\begin{array}{l}\text { Between six months and } \\
\text { one year }\end{array}$ & $35(17.24 \%)$ \\
\hline $\begin{array}{l}\text { Between one year and } \\
\text { five years }\end{array}$ & $32(15.76 \%)$ \\
\hline More than five years & $11(5.41 \%)$ \\
\hline $\begin{array}{l}\text { Unknown (no symptoms } \\
\text { present) }\end{array}$ & $53(26.10 \%)$ \\
\hline
\end{tabular}

\begin{tabular}{|l|l|}
\hline Buccal mucosa & $53(26.10 \%)$ \\
\hline Tongue & $13(6.40))$ \\
\hline Gingiva & $8(3.94 \%)$ \\
\hline $\begin{array}{l}\text { Buccal mucosa and } \\
\text { tongue }\end{array}$ & $52(25.61 \%)$ \\
\hline $\begin{array}{l}\text { Buccal mucosa and } \\
\text { gingiva }\end{array}$ & $10(4.92 \%)$ \\
\hline Other locations & $67(33 \%)$ \\
\hline
\end{tabular}

TABLE 1. Main characteristics of patients including the medical history, anamnestic data, and clinical features of the OLP patients 
TABLE 2. The clinical forms of oral lichen planus according to the presence of candidiasis infection

\begin{tabular}{|l|l|l|}
\hline Clinical form & $\begin{array}{l}\text { Candida positive (no and } \\
\text { percentage of patients) }\end{array}$ & $\begin{array}{l}\text { Candida negative (no and } \\
\text { percentage of patients) }\end{array}$ \\
\hline Keratosis OLP & $22(29.33 \%)$ & $47(36.71 \%)$ \\
\hline Atrophic OLP & $33(44 \%)$ & $47(36.71 \%)$ \\
\hline Erosive-ulcerative OLP & $17(22.66 \%)$ & $27(21.09 \%)$ \\
\hline Bullous OLP & $3(4 \%)$ & $7(5.46 \%)$ \\
\hline Total & 75 & 128 \\
\hline
\end{tabular}

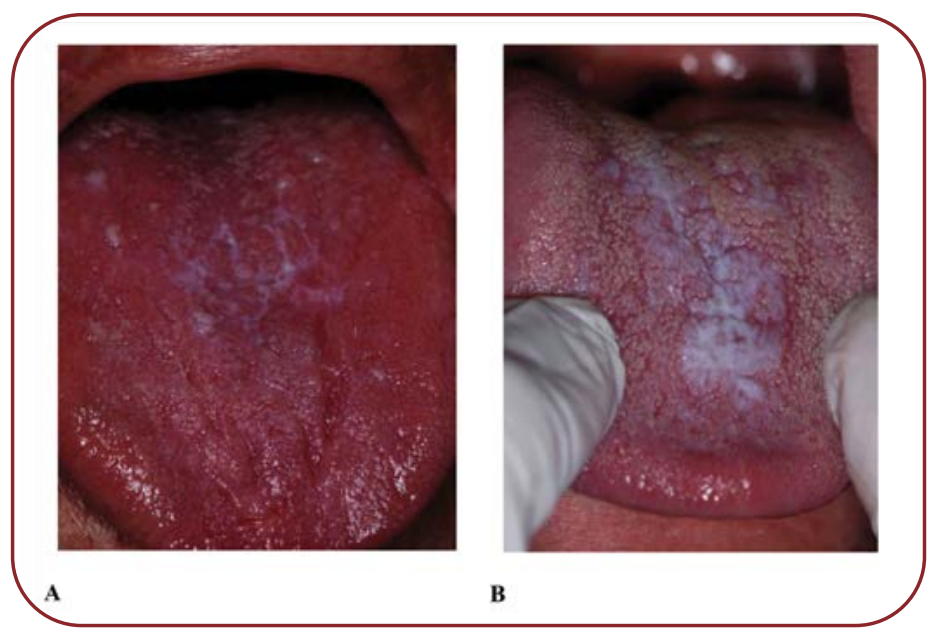

FIGURE 1. Oral keratotic lichen planus of the dorsal tongue with Candida infection (A) and without Candida infection (B)

common in 64 cases (85.33\%). Other non-Candida albicans species detected were Candida krusei four cases, Candida lusitaniae and Candida tropicalis two cases each (2.66\%) and Candida glabrata, Candida kefyr, and Candida sphaerica, one case each (1.33\%).

The association between the clinical forms and presence of Candida infection was not statistically significant for any clinical form $(p>0.05$, Fisher test).

\section{DISCUSSION}

Eor a long time, the connection between Candida infection and chronic oral mucosal diseases, especially OLP and oral leukoplakia, has received increasing attention from oral medicine practitioners, dentists, and general practitioners.
The aim of this study was to investigate the presence and species of Candida in OLP lesions and how this impacts its clinical features.

A recent review from a microbiological point of view concerning OLP reinforces the idea that this autoimmune disease can provide favorable circumstances for microbial colonization (11). He et al suggested that OLP may predispose to certain specific genotypes of Candida (12).

As previously observed in most OLP studies $(2,3)$, in the present study, this disease is more common in women $(75.86 \%$ females vs $24.13 \%$ males). Patients were mainly addressed for burning sensation (33.99\%) and most of them (34.48\%) in the first six months from the onset of lesions. Buccal mucosa was the most frequently affected site of the oral mucosa, which is in concordance with previously reported studies $(2,3)$.

In the present study, we detected oral candidiasis associated with OLP in $36.94 \%$ of patients, which is in the range of previous studies $11 \%$ to $47 \%$ (13). Oral candidiasis added to the OLP lesions usually aggravates the symptoms and the clinical features.

The current study results did not show any statistically significant differences between clinical patterns of OLP and Candida infection. In atrophic and erosive forms of OLP, the presence of candidiasis can worsen the lesions mainly correlated to the inflammatory response caused by adherence to the oral mucosa (14). Such cases were also encountered in our clinical experience. But the identification of Candida species is important for successful therapy selection and later OLP clinical evolution.

As OLP has a chronic evolution, in most cases, complete clinical remission is difficult to achieve even under treatment. The mainly used standard topical treatment is a moderate corticosteroid integrated into Orabase, which can favor Candida growth and recurrences of oral candidiasis. The previously reported incidence of $13.6 \%$ of oral candidiasis in steroid treated OLP patients emphasizes the importance of antifungal adjuvant treatment (15). The OLP treatment periods, number of topical applications, and recommendations for antifungal use are individualized for each patient depending on lesion severity and general health status. The association of general diseases or different physiological conditions imposes the need for implications from other medical specialties. For example, in OLP 
associated with pregnancy it is recommended to avoid treatment. Still, in severe erosive cases, pregnant women may accept treatment after prior consultation with the obstetrician to assess fetal risks (16).

Besides oral candidiasis, some other local factors causing prolonged healing of OLP lesions are inefficient oral hygiene and dental foci. Moreover, although probiotic bacteria were reported to reduce the oral Candida carriage in vivo in OLP lesions, this treatment did not reduce the recurrence of oral candidiasis (17).

\section{CONCLUSIONS}

$\int$ the espite the small sample size of our study and the fact that no significant difference was found between clinical features of OLP with and without superimposed oral candidiasis, both oral diseases were present in $36.94 \%$ of patients. In our experience, routine screening for oral candidiasis and a preventive antifungal strategy in OLP patients improves the evolution of oral lesions. $\square$

Conflicts of interest: none declared. Financial support: none declared.

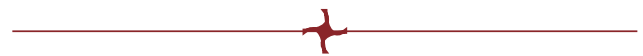

\section{$\mathbf{R}_{\text {EFERENCES }}$}

1. Țovaru S, Ţovaru M, Costache $M$, Demarosi F, Mihai L. Medicină şi patologie orală, vol. I, QMed Publishing Press, București, 2012

2. Changchang Li, Xiaoqiong Tang, Xiaoyan Zheng, et al. Global prevalence and incidence estimates of oral lichen planus: a systematic review and meta-analysis. JAMA Dermatology 2020;156:172-181.

3. Scully C, Carrozzo M. Oral mucosal disease: Lichen planus. Br J Oral Maxillofac Surg 2008;46:15-21.

4. Boñar-Alvarez $P$, Pérez Sayáns $M$, Garcia-Garcia A, et al. Correlation between clinical and pathological features of oral lichen planus: A retrospective observational study. Medicine 2019;98:e14614.

5. Van der Meij EH, Van der Waal I. Lack of clinicopathologic correlation in the diagnosis of oral lichen planus based on the presently available diagnostic criteria and suggestions for modifications. Journal of Oral Pathology \& Medicine 2003;32:507-512.

6. Gheorghe C, Mihai L, Parlatescu I, Tovaru S. Association of oral lichen planus with chronic $C$ hepatitis. Review of the data in literature. Maedica 2014;9:98-103.

7. Shen ZY, Liu W, Feng JQ, et al. Squamous cell carcinoma development in previously diagnosed oral lichen planus: de novo or transformation? Oral Surgery, Oral Medicine, Oral Pathology, Oral Radiology, and Endodontology 2011;112:592-596.

8. Williams A, Williams D, Rogers H, et al. Immunohistochemical Expression Patterns of Inflammatory Cells Involved in Chronic Hyperplastic Candidosis. Pathogens 2019;8:232.

9. Kragelund C, Kieffer-Kristensen L, Reibel J, et al. Oral candidosis in lichen planus: the diagnostic approach is of major therapeutic importance. Clinical Oral Investigations 2013;17:957-965

10. Parlatescu I, Radu L, Nicolae C. Analysis of Candida Infection Impact in Oral Lichen Planus Patients. Maedica-a Journal of Clinical Medicine 2020;15 Supp.

11. Villa TG, Sánchez-Pérez Á, Sieiro C. Oral lichen planus: a microbiologist point of view.

International Microbiology 2021;24:275-289.

12. He $H, X i a X$, et al. A pilot study: a possible implication of Candida as an etiologically endogenous pathogen for oral lichen planus.

BMC Oral Health 2020;20:1-8.

13. Miranda F, Werneck JT, Dias EP, et al. Clinical Behavior of Oral Lichen Planus in Association with Candidiasis. Medical Mycology: Open Access 2020;7.

14. Lundstrom IM, Anneroth GB, Holmberg K. Candida in patients with oral lichen planus. Int J Oral Surg 1984;13:226-238.

15. Marable DR, Bowers LM, Stout TL, et al. Oral candidiasis following steroid therapy for oral lichen planus. Oral Dis 2016;22:140-147.

16. Wei J, Ma D, Qiu M, et al. Medical treatments for pregnant patients with oral lichen planus. Acta Odontol Scand 2017;75:67-72.

17. Keller MK, Kragelund C. Randomized pilot study on probiotic effects on recurrent candidiasis in oral lichen planus patients. Oral Dis 2018;24:1107-1114. 